\title{
Influence of the Anisometry of Magnetic Particles on the Isotropic-Nematic Phase Transition
}

\author{
V. Gdovinováa ${ }^{2}$ N. Tomašovičováa*, N. Éber ${ }^{b}$, T. \\ Tóth-Katona $^{\mathrm{b}}$, V. Závišováa ${ }^{\mathrm{a}}, \mathrm{M}$. Timko ${ }^{\mathrm{a}}$ and P. Kopčanskýa \\ ${ }^{a}$ Institute of Experimental Physics, Slovak Academy of Sciences, Watsonová 47, 04001 \\ Košice, Slovakia; ${ }^{b}$ Institute for Solid State Physics and Optics, Wigner Research Centre for \\ Physics, Hungarian Academy of Sciences, H-1525 Budapest, P.O.Box 49, Hungary
}

\begin{abstract}
The influence of the shape anisotropy of magnetic particles on the isotropic-nematic phase transition was studied in ferronematics based on the nematic liquid crystal 4-(trans-4-n-hexylcyclohexyl)isothiocyanato-benzene (6CHBT). The liquid crystal was doped with spherical or rod-like magnetic particles of different size and volume concentrations. The phase transition from isotropic to nematic phase was observed by polarizing microscope as well as by capacitance measurements. The influence of the concentration and the shape anisotropy of the magnetic particles on the isotropicnematic phase transition in liquid crystal is demonstrated. The results are in a good agreement with recent theoretical predictions.
\end{abstract}

Keywords: liquid crystal; magnetic nanoparticles; ferronematics; anisotropy 


\section{INTRODUCTION}

Doping liquid crystals (LCs) with nanoparticles (NPs) in low volume concentrations has been shown as a promising method to modify the properties of liquid crystals. The presence of nanoparticles in liquid crystal changes the properties of the mesophase and/or introduces some new features of the composite mixtures. After introducing the idea theoretically by Brochard and de Gennes [1] several reports have shown that the doping of liquid crystals with magnetic nanoparticles can either increase or decrease the critical field of the magnetic Fréedericksz transition [2 5], depending on the host-guest combination. It has also been proven [6, 7] that ferronematics respond to low magnetic fields (below $0.1 \mathrm{~T}$ ) due to doping with magnetic particles. Furthermore, the theoretically predicted magnetic field-induced isotropic-nematic phase transition [8] was observed in calamitic liquid crystals at relatively low magnetic fields (below $12 \mathrm{~T}$ ) when they are doped with magnetic nanoparticles [9].

Properties of magnetic nanoparticles significantly depend on their size, shape and structure. Therefore, the properties of ferronematics composed of them are also expected to be sensitive to the same factors. As an example, in our previous work [2] it was shown that doping the liquid crystal 4-(trans-4-n-hexylcyclohexyl)-isothiocyanato-benzene (6CHBT) with spherical magnetic particles results in soft anchoring $(\omega \sim 1)$, while in the case of doping with rod-like magnetic particles rigid anchoring $\left(\omega \sim 10^{4}\right)$ was obtained at the LC-NP interface, even though both kinds of magnetic particles were coated with the same surfactant (oleic acid). Here, the parameter $\omega=W R / K$ is the ratio of the interfacial (anchoring) to the bulk (elastic) energies; $W$ is the anchoring energy density, $R$ is the reference size of the

NPs [10] which in case of cylindrical particles coincides with their mean radius $D / 2$ [10, 11], and $K$ is the corresponding elastic modulus of the host LC.

Recently, a mean-field theory has been developed by Gorkunov and Osipov [12] to describe the influence of embedded nanoparticles on the orientational order and on the isotropicnematic phase transition of the host liquid crystal. They considered relatively large, uniaxial NPs of oblate or prolate shape, where the interaction between the LC molecules and the NPs may be described by an anisotropic surface potential depending on the coupling between the surface normal of the particle and the long molecular axis of the host. It has been shown that spherically isotropic nanoparticles effectively dilute the liquid crystal medium and decrease the isotropic-nematic transition temperature. On the contrary, anisotropic nanoparticles 
become aligned by the nematic host and, reciprocally, improve the liquid crystal alignment, thus extending the nematic order to higher temperatures.

This theory has inspired us to perform measurements with the nematic liquid crystal 6CHBT doped with spherical or rod-shaped magnetic nanoparticles. The present work is devoted to an experimental study of how the shape, as well as the volume concentration of magnetic nanoparticles affect the temperature of the isotropic-nematic phase transition. The obtained results indicate a significant effect in accordance with the theoretical expectations described in [12].

\section{EXPERIMENT}

The spherical magnetic NPs were prepared by the co-precipitation method described in 2]. The rod-like iron oxide NPs were synthesized through smooth decomposition of urea [5]. The smaller particles were synthesized by co-precipitation of $\mathrm{Fe}^{3+}$ and $\mathrm{Fe}^{2+}$ in oleic acid micelles. The synthesis of the longer iron oxide particles utilized precipitation of $\mathrm{Fe}^{2+}$ in the presence of oleic acid as stabilizer. The morphology and size distribution of the prepared NPs were determined by transmission electron microscopy (TEM). Fig. 1 shows the TEM images of the prepared spherical and rod-like nanoparticles. The mean diameter

of the spherical magnetic NPs was $D=10 \mathrm{~nm}$. The average diameter of the shorter rod-like nanoparticles was $D=8 \mathrm{~nm}$ and the mean length was $L=40 \mathrm{~nm}$. The longer rod-like NPs had the average diameter of $D=11 \mathrm{~nm}$ and their mean length was $L=240 \mathrm{~nm}$.

The ferronematic samples were based on the thermotropic nematic 6CHBT which is a low-temperature-melting enantiotropic liquid crystal with high chemical stability [13]. The phase transition temperature from the isotropic liquid to the nematic phase (the clearing point) of the studied nematic was found by polarizing optical microscopy at $\mathrm{T}_{I N}=43.3^{\circ} \mathrm{C}$. The doping was done by adding nanoparticles (spherical or rod-like) to the liquid crystal in the isotropic phase under continuous stirring. The nanoparticles were coated with oleic acid as a surfactant to suppress their aggregation. The ferronematic samples were prepared with three different volume concentrations of the spherical as well as of the rod-like magnetic particles: $\phi_{1}=1 \times 10^{-5}, \phi_{2}=5 \times 10^{-5}$ and $\phi_{3}=1 \times 10^{-4}$.

The structural transition from the isotropic to the nematic phase was monitored by polarizing microscope as well as by capacitance measurements. The prepared samples were filled 
into a capacitor made of ITO-coated glass electrodes with the electrode area approximately $1 \mathrm{~cm} \times 1 \mathrm{~cm}$. The distance between the electrodes (the sample thickness) was $d=5 \mu \mathrm{m}$. The samples (the undoped 6CHBT, or 6CHBT doped by various NPs) were filled into the cells in the isotropic phase due to capillary forces. A rubbed polyimide coating on the electrodes ensured planar orientation.

For all samples the measurements started with polarizing microscopic observations. The samples were put into a Linkam hot stage, heated to the isotropic state, then the samples were slowly cooled (at the rate of $1^{\circ} \mathrm{C} / \mathrm{min}$ ) to the nematic state while monitoring their textures between crossed polarizers. The transition temperature $\mathrm{T}_{I N}$ was taken as the temperature, where nematic droplets appeared in the isotropic melt in the cooling process. Next, the temperature dependence of the capacitance of the same samples was measured in the same cells at the frequency of $1 \mathrm{kHz}$ by a high precision capacitance bridge Andeen Hagerling (the accuracy at $1 \mathrm{kHz}$ is $0.8 \mathrm{aF}$ ). The samples were first heated to the isotropic phase and then they were again slowly cooled to the nematic phase (at the rate of $1^{\circ} \mathrm{C} / \mathrm{min}$ ).

\section{RESULTS AND DISCUSSION}

Fig. 2 shows the temperature dependence of the reduced capacitance $\left(C-C_{\max }\right) /\left(C_{\max }-\right.$ $C_{\text {min }}$ ) for the undoped 6CHBT and for the ferronematics containing spherical magnetic NPs in different volume concentrations. Here $C, C_{\max }$ and $C_{\min }$ correspond to the capacitances at the actual, at the highest and at the lowest temperatures, respectively. Each curve exhibits a monotonic decrease of the capacitance with diminishing temperature. In the isotropic phase the capacitance is nearly constant. The sudden drop of the capacitance indicates the appearance of the orientational order (and hence the increase of the anisotropy), i.e. the phase transition to the nematic phase. The decrease of $C$ occurs due to the planar alignment and the positive dielectric anisotropy of 6CHBT and the 6CHBT-based ferronematics. Fig. 2 clearly shows that doping with spherical NPs results in a shift of $T_{I N}$ towards lower temperatures; the shift becomes larger with increasing the volume concentration of magnetic NPs. This is in accordance with the expectations, as NPs behave as impurities introducing disorder and thus reducing $T_{I N}$. Figs. 3 and 4 also show the temperature dependence of the reduced capacitance, however, for ferronematics doped by short and long rod-like magnetic nanoparticles, respectively. The monotonicity of the temperature dependence and the easy 
detectability of the isotropic-nematic phase transition temperature hold here too. However, in the case of doping 6 CHBT with rod-like magnetic NPs, $T_{I N}$ shifts towards higher temperatures. Moreover, though the increasing volume concentration of NPs initially enhances the shift, this tendency turns over for higher concentration. Nevertheless, $T_{I N}$ still remains higher for the samples doped with rod-like NPs than that in the undoped LC.

The differences between the behaviour of ferronematics containing NPs of different shape is even more perceptible in Fig. 5 which shows the dependence of the $T_{I N}$ on the volume concentration of NPs, obtained by capacitance measurements and by polarizing microscopy. As one can see, the results provided by the two independent techniques are in good agreement. Fig. 5 clearly shows, that the increase of the phase transition temperature is more pronounced when long rod-like NPs are used for the doping instead of the short ones. In order to understand these features one has to recall the recent theory of Gorkunov and Osipov [12]. They have developed a mean-field molecular description of the thermodynamics of nematic liquid crystals mixed with NPs, which may be anisotropic due to their shape, their surface treatment, and/or their spontaneous polarization. As it has been shown, the large shape anisotropy of NPs (namely the rod-likeness) enhances the nematic order and, as a consequence, increases the phase transition temperature. In contrast to that, spherical NPs reduce the orientational order and thus lower $T_{I N}$. These conclusions are in agreement with our experimental findings presented in Figs. 2 - 5 and explain the different signs of the shift of $T_{I N}$ for spherical and rod-like NPs. Moreover, it is evident from our TEM measurements that longer rod-like nanoparticles have a larger length/diameter ratio $(\approx 22)$ than the shorter NPs $(\approx 5)$; i.e. longer NPs have higher anisotropy and thus larger shift in $T_{I N}$.

In order to prevent aggregation of NPs, they are usually coated with surfactants during the preparation. In a recent work various ligands were used for this purpose for two different NPs of spherical shape dispersed in a polymorphic LC [14]. A slight, though measurable change in the phase transition temperatures has been reported in these dispersions. However, the influence of the type and concentration of the NPs, as well as the type of the surfactant on the phase transition temperature shift could not be distinguished clearly from these measurements. Therefore, we have coated all magnetic NPs (spherical and rod-like) with the same surfactant (oleic acid). The theoretical description in [12] remains valid if the surface of the nanoparticles is coated by organic molecules. However, one has to take into account that in general, the surfactant dilutes the liquid crystal, reduces its order and thus 
lowers the isotropic-nematic phase transition temperature in itself. In the case of doping with coated magnetic nanoparticles, the increasing volume concentration of NPs increases the concentration of the surfactant as well. When spherical NPs are used as dopants, the surfactant and the NPs have a similar effect on $T_{I N}$ resulting in its decrease, as shown in Fig. 2. In contrast to that, using rod-like dopants the NPs and the surfactant counteract; the effect of the surfactant (which decreases $T_{I N}$ ) seems to prevail at higher concentrations. This may explain the nonmonotonic concentration dependence of $T_{I N}$ seen in Fig. 5, and is also in good qualitative agreement with the Gorkunov-Osipov theory.

Finally, we note that in ferronematics based on 6CHBT, the magnetic moment (which is in case of rod-like NPs parallel with their long axis) coincides with the nematic director as it has been shown in our previous work [2]. However, because of the low volume concentration (order of $10^{-5}-10^{-4}$ ) and small value of the magnetic moment (order of $10^{-24} \mathrm{Tm}^{3}$ ) of NPs their magnetic interaction can be considered negligible, and therefore, does not influence the LC order significantly.

\section{CONCLUSION}

We have found that the shape as well as the volume concentration of magnetic nanoparticles have a significant influence on the temperature of the isotropic to nematic phase transition of ferronematics. We have justified that ferronematics doped with rod-like magnetic nanoparticles have higher $T_{I N}$ than the host nematic or ferronematics containing spherical nanoparticles. We have found that doping with rod-like NPs yields a nonmonotonic concentration dependence of $T_{I N}$, which could be attributed to the competing influence of the nanoparticles and of the organic surfactants they are coated with. Our results provide a firm experimental proof for the main conclusion of the recent mean-field theory of Gorkunov and Osipov [12].

\section{Acknowledgments}

This work was supported by project VEGA 0045, the Slovak Research and Development Agency under the contract No. APVV-0171-10, Ministry of Education Agency for Structural Funds of EU in frame of projects 6220120021 and 6220120033, the Hungarian Research Fund 
OTKA K81250 and M-era.Net project MACOSYS (OTKA NN110672).

\section{REFERENCES}

[1] Brochard F, de Gennes PG. Theory of magnetic suspensions in liquid crystals. J. Phys. (Paris) 1970;31:691-708.

[2] Kopčanský P, Tomašovičová N, Koneracká M, Závišová V, Timko M, Džarová A, Šprincová A, Éber N, Fodor-Csorba K, Tóth-Katona T, Vajda A, Jadzyn J. Structural changes in the 6CHBT liquid crystal doped with spherical, rodlike, and chainlike magnetic particles. Phys. Rev. E. 2008;78:011702.

[3] Kopčanský P, Tomašovičová N, Koneracká M, Timko M, Závišová V, Éber N, Fodor-Csorba K, Tóth-Katona T, Vajda A, Jadzyn J, Beaugnon E, Chaud E. The structural instabilities in ferronematics based on liquid crystal with negative diamagnetic susceptibility anisotropy. J. Magn. Magn. Mater. 2010;322:3696-3700.

[4] Podoliak N, Buchnev O, Bavkin DV, Kulak AN, Kaczmarek M, Sluckin TJ. Magnetite nanorod thermotropic liquid crystal colloids; synthesis, optics and theory. J. Colloid Interface Sci. $2012 ; 386: 158-166$.

[5] Tomašovičová N, Kopčanský P, Éber N. Magnetically active anisotropic fluids based on liquid crystals. In: Lemu HG, editor. Anisotropy research: new developments. Chapter 11. Hauppauge (NY): Nova Science Publishers; 2012. p. 245-276.

[6] Podoliak N, Buchnev O, Buluy O, D'Alessandro G, Kaczmarek M, Reznikov Y, Sluckin TJ. Macroscopic optical effect in low concentration ferronematics. Soft Matter. 2011;7:4742-4749.

[7] Tomašovičová N, Timko M, Mitróová Z, Koneracká M, Rajňak M, Éber N, Tóth-Katona T, Cahud X, Jadzyn J, Kopčanský P. Capacitance changes in ferronematic liquid crystals induced by low magnetic fields. Phys. Rev. E. 2013;87:014501.

[8] Lelidis I, Durand G. Electric-field-induced isotropic-nematic phase transition. Phys. Rev. E. $1993 ; 48: 3822-3824$.

[9] Kopčanský P, Tomašovičová N, Koneracká M, Závišová V, Timko M, Hnatič M, Éber N, TóthKatona T, Jadzyn J, Honkonen J, Beaugnon E, Chaud X. Magnetic-field induced isotropic to 
nematic phase transition in ferronematics. IEEE Trans. Magn. 2011;47:4409-4412.

[10] Burylov SV, Raikher YL. Macroscopic properties of ferronematics caused by orientational interactions on the particle surfaces. I. Extended continuum model. Mol. Cryst. Liq. Cryst. $1995 ; 258: 107-122$.

[11] Burylov SV, Raikher YL. Orientation of a solid particle embedded in a monodomain nematic liquid crystal. Phys. Rev. E. 1994;50:358-367.

[12] Gorkunov MV, Osipov MA. Mean-field theory of a nematic liquid crystal doped with anisotropic nanoparticles. Soft Matter. 2011;7:4348-4356.

[13] Dabrowski R, Dziaduszek J, Szczucinski T. 4-(Trans-4-n-Alkylcyclohexyl) Isothiocyanatobenzenes a new class of low-melting stable nematics. Mol. Cryst. Liq. Cryst. Lett. 1984;102:155160.

[14] Prodanov MF, Kolosov MA, Krivoshey AI, Fedoryako AP, Yarmolenko SN, Semynozhenko VP, Goodby JW, Vashchenko VV. Dispersion of magnetic nanoparticles in a polymorphic liquid crystal. Liq. Cryst. 2012;39(12):1512-1526. 


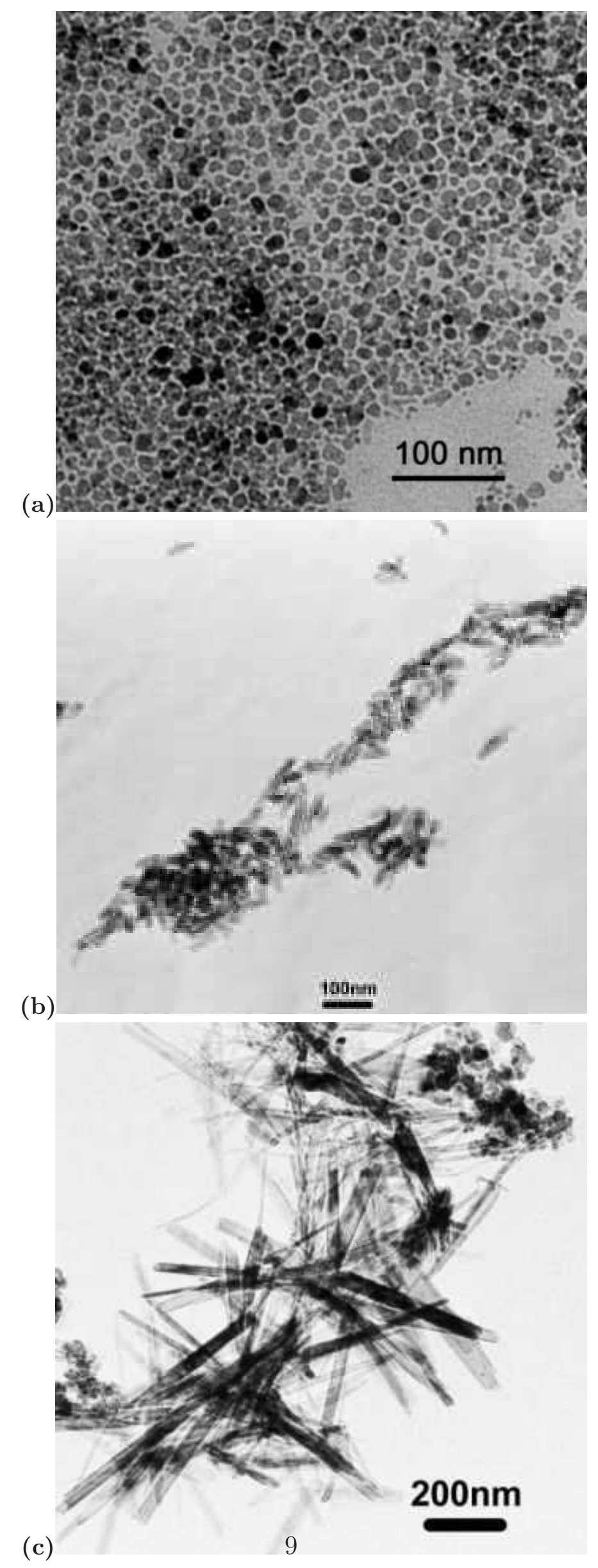




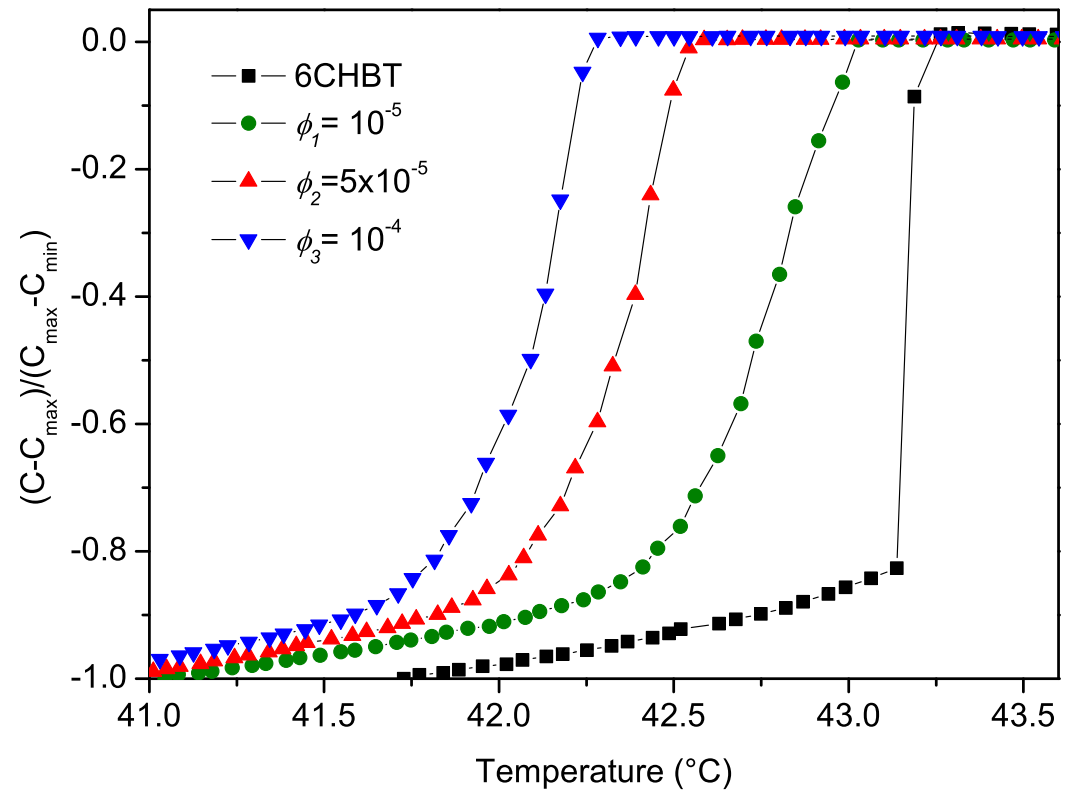

FIG. 2: Reduced capacitance vs. temperature for undoped 6CHBT and for 6CHBT doped with spherical magnetic nanoparticles of different concentrations $\phi$. 


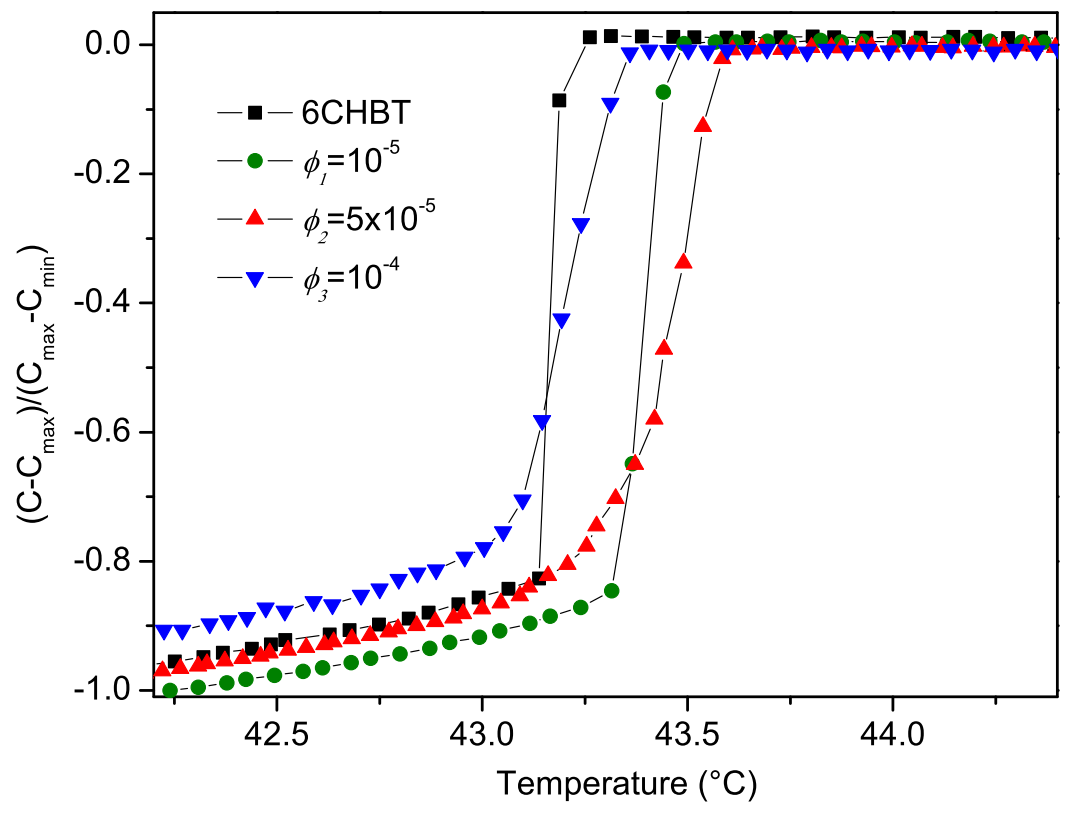

FIG. 3: Reduced capacitance vs. temperature for undoped 6CHBT and for 6CHBT doped with short rod-like magnetic nanoparticles of different concentrations $\phi$. 


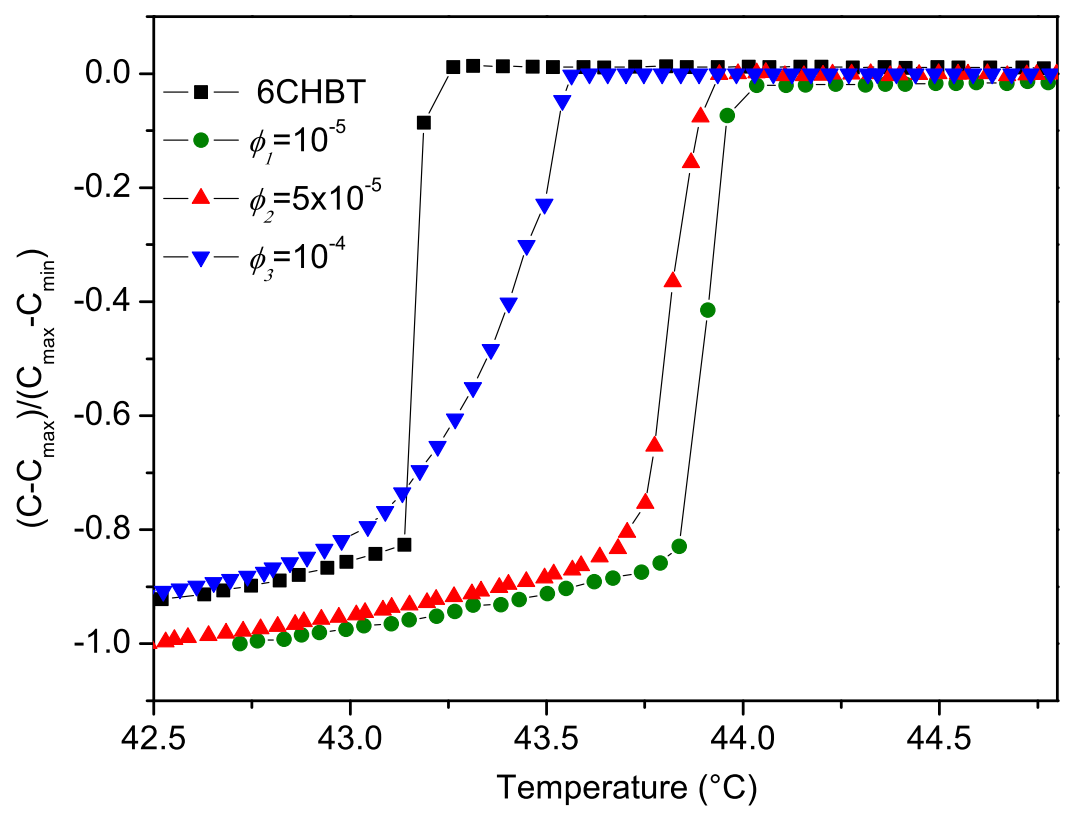

FIG. 4: Reduced capacitance vs. temperature for undoped 6CHBT and for 6CHBT doped with long rod-like magnetic nanoparticles of different concentrations $\phi$. 


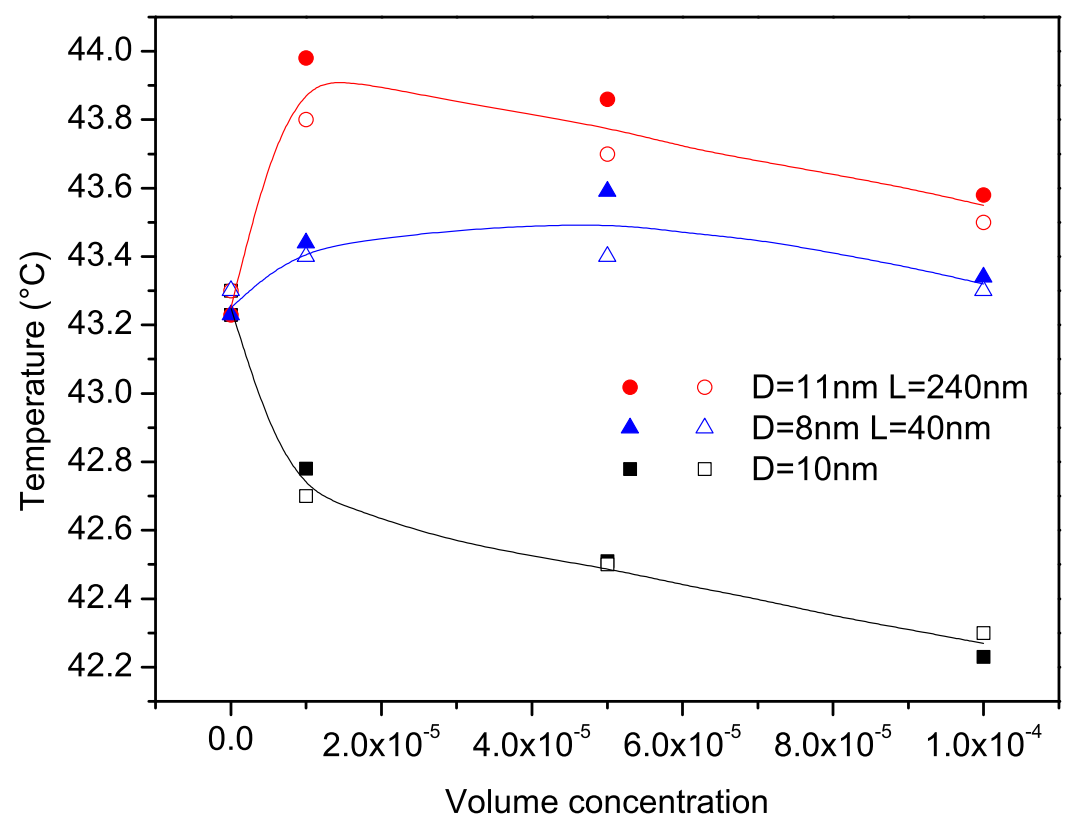

FIG. 5: Dependence of the isotropic to nematic phase transition temperature on the volume concentration for spherical and rod-like magnetic nanoparticles (as indicated in the legend), obtained by capacitance measurements (full symbols) and determined by polarizing microscopy (open symbols). The solid lines are guides to the eye. 